\title{
Impact of symmetry on ergodic properties of triangular billiards
}

\author{
Katerina Zahradova, ${ }^{*}$ Julia Slipantschuk, Oscar F. Bandtlow, and Wolfram Just \\ School of Mathematical Sciences, Queen Mary University of London, Mile End Road, London E1 4 NS, UK
}

(Dated: November 18th, 2021)

\begin{abstract}
Polygonal billiards constitute some of the simplest yet counterintuitive dynamical systems in physics. Even basic features of the dynamics, such as ergodicity of the microcanonical distribution or the decay of correlations have not been settled in general. In this numerical study, we will highlight the importance of symmetries of the billiard table for the resulting dynamics. While typical triangular billiards appear to show correlation decay, symmetric billiards may not even be ergodic with respect to the uniform distribution in phase space.
\end{abstract}

PACS numbers: 05.45.-a, 05.20.-y, 05.70.Fh

Keywords: Billiards, Ergodicity, Mixing

Introduction The study of complex dynamical behaviour is one of the most vibrant areas of research at the interface of mathematics, theoretical physics, and their application to real world phenomena. While challenges remain, the basic mechanisms for chaotic dynamics such as sensitivity, hyperbolicity, and correlation decay have by now been identified, see e.g., [1-4]. The next frontier of understanding in dynamical systems theory thus lies in systems without uniform hyperbolicity or without exponential decay of correlations (typically, parabolic systems), an area sometimes referred to as anomalous dynamics, see e.g., $[5,6]$ for typical references covering the wide range from rigorous mathematical approaches to real world applications. Polygonal billiards are the simplest prototypes of such systems [7], with the mechanisms for the creation of sensitivity or irregular motion poorly understood to date.

There is a substantial body of mathematical literature, in particular for rational billiards, where angles between sides are rational multiples of $\pi$. The wealth of knowledge about rational billiards is due to the possibility of invoking the machinery of interval exchange transformations, which makes it possible to develop computable criteria for various dynamical properties such as minimality [8], ergodicity [9], or weak mixing [10], while (strong) mixing and even the occurrence of a mixing factor can be excluded by the seminal result [11]. Polygonal billiards which are weakly mixing have been described in [12]. To the best of our knowledge, the only result concerning general polygonal billiards is the ergodicity of Lebesgue measure for billiard tables that are typical in a certain sense [13]. The key method used here involves sophisticated approximations of general polygons by polygons with angles which are rational multiples of $\pi$. A constructive example can be found in [14]. For accessible reviews giving further insight into this fascinating field see, e.g., [15-17].

Due to the limited mathematical progress for irrational billiards the analysis in the physics literature has been entirely based on numerical simulations, see e.g. [18, 19]. Numerical results indicate that irrational billiards are er- godic with respect to Lebesgue measure, while the correlation decay indicates weak and even strong mixing, see $[20,21]$. However, as pointed out recently [22] the numerical results are not fully conclusive. Here we revisit this strand of research and point out another facet of this problem, namely the role of symmetry. In the absence of a suitable mathematical machinery we will resort to an extensive numerical analysis. While our analysis may not be fully conclusive, our results point towards a surprisingly rich dynamical structure, given that the underlying dynamics looks almost trivial. On the one hand our analysis reinforces the belief that typical irrational asymmetric billiards are ergodic and mixing. On the other hand ergodicity seems to be questionable for symmetric billiards.

Mixing in general asymmetric triangles The renewed interest in general billiards with irrational angles was triggered by [18, 19], which provided numerical evidence for correlation decay in systems without an obvious mechanism like sensitivity. This makes polygonal billiards one of the most challenging mathematical and theoretical subjects of our time.

To begin, we revisit this setup. We consider a triangle with inner angles $\alpha, \beta$, and $\pi-\alpha-\beta$ and we focus on the generic case with $\alpha / \pi$ and $\beta / \pi$ irrational, and all angles distinct. For the purpose of our simulation we take $\alpha=\pi(\sqrt{2}-1) / 4$ and $\beta=\pi(\sqrt{5}-1) / 4$ but the results quoted below do not seem to depend substantially on these particular values. To capture the dynamics we consider the billiard map $T$ which gives the relation between two subsequent bounces with the boundary. As usual we use so-called Birkhoff coordinates $(s, p)$ where $s$ denotes the position of the bounce measured in terms of the arclength along the boundary and $p=\cos (\phi)$ is the velocity component of the outgoing ray, tangential to the boundary, while assuming that the particle moves with unit speed. Using these coordinates the billiard map is area preserving so that Lebesgue measure in phase space constitutes an invariant measure of the system.

The quantity of interest is the autocorrelation function 
of an observable $f$ which is given by

$$
C_{f f}(n)=\left\langle f \cdot f \circ T^{n}\right\rangle_{\mu}-\langle f\rangle_{\mu}^{2}
$$

where $\langle\ldots\rangle_{\mu}$ denotes the average with respect to an invariant measure $\mu$. Based on rigorous results for rational billiards, and lacking an obvious mechanism, one would not expect the correlation function to decay, i.e., the billiard map to be mixing, see e.g. [15]. The best one could hope for is weak mixing, see [11], implying that correlation functions do not tend to zero, while their absolute Cesaro sum $\sum_{n=0}^{N-1}\left|C_{f f}(n)\right| / N$ tends to zero as $N \rightarrow \infty$. Hence, the results of $[18,19]$ came as a slight surprise as the numerical simulations appeared to indicate that correlations may decay.

For the numerical computation of the correlation function we use a standard FFT approach. We chop a long time series into shorter pieces, use a Fourier transform and the Wiener-Khinchin theorem to compute the autocorrelation and finally take the ensemble average over all the pieces. In between we discount the zero frequency component to account for the autocovariance. This approach is able to cope with cases where the invariant density is not known a priori, as the ensemble average, based on a time series, realises the physical invariant measure. For the billiard map considered here, the result of this approach (see Fig. 1) is numerically identical to a computation using initial conditions sampled uniformly at random, thus providing further evidence for the ergodicity of Lebesgue measure.

Mixing requires correlations to decay for all square integrable observables $f$. In simulations one can only check very few observables, and one often insinuates that the findings are generic. In our case we have checked a few observables, involving arclength $s$ and momentum $p$, all revealing essentially the same properties.

The correlations of $p$ (see Fig. 1) show a power law decay with a levelling off at large time scales. The large time plateau value scales with the ensemble size consistent with that for sums of independent random numbers (see inset in Fig. 1). Hence there is compelling evidence that this levelling off is caused by sampling errors due to finite sample size. For the setup of Fig. 1 we observe a power law decay for $C_{p p}(n)$ with exponent -1.4 . Correlations for a range of other irrational triangles and observables show the same qualitative behaviour, but the exponent reveals a weak dependence on the observable and a considerable dependence on the angles of the triangle.

The properties shown in Fig. 1 are typical for simulations of a larger class of observables and triangles. No major number theoretic impact of the irrational angle values is visible, and the simulations give support for mixing in asymmetric generic irrational triangles. In addition, the same feature can be found in simple model maps which have been proposed to exhibit properties of billiard maps [20], see also [21]. Unfortunately, no basic

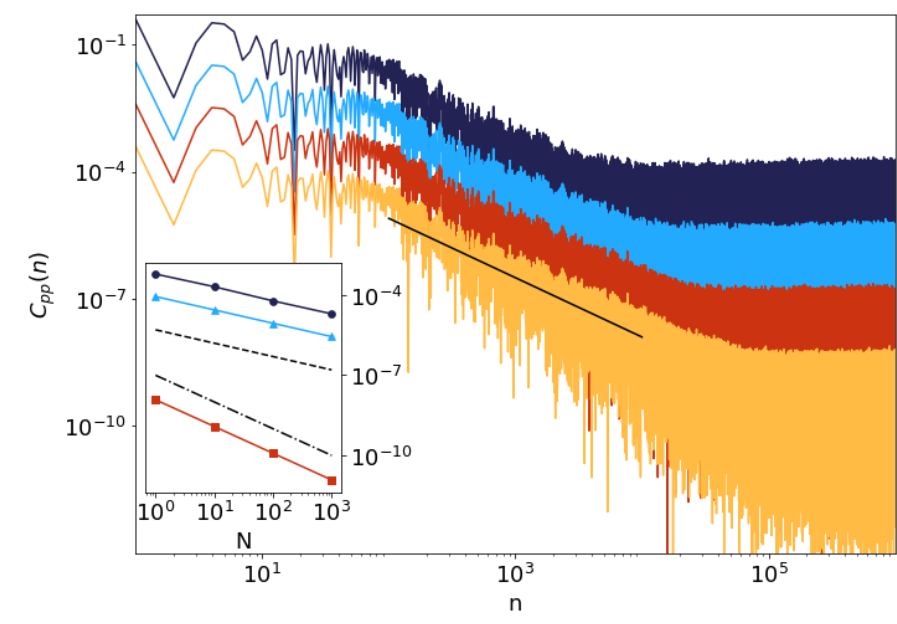

FIG. 1. Autocorrelation function of the momentum $p$ on a double logarithmic scale for an asymmetric billiard with inner angles $\alpha=\pi(\sqrt{2}-1) / 4$ and $\beta=\pi(\sqrt{5}-1) / 4$. Data have been computed from a time series of length $2^{30}$ with ensembles of initial conditions of different sizes: dark blue (top) $N=1$, light blue (second from top) $N=10$, red (third from top) $N=$ 100, yellow (bottom) $N=1000$. For visibility, values have been shifted by a factor of $10^{-1}$. The solid black line indicates a power law decay with exponent -1.4 . The inset displays the dependence of the plateau for large time as a function of the ensemble size, measured in terms of the maximum (dark blue circles), the absolute mean (light blue triangles), and the variance (red squares). The additional black lines indicate a power law decay with exponents $1 / 2$ (dashed) and 1 (dashdotted).

mechanism, let alone a mathematical approach, has been identified so far to put the conjecture of correlation decay in typical triangular billiards on a firm basis.

Ergodicity breaking in isosceles triangles By the seminal result of [13], Lebesgue measure is ergodic for triangular billiards for a large set of angles, when the property being a large set is measured in topological terms. As indicated by [18] and the results of the preceding section, this in fact seems to hold in typical numerical simulations. However one has to recall that the question of ergodicity of typical polygonal billiards is largely unsolved, as, e.g., it is unclear whether the set of ergodic billiards has positive Lebesgue measure [7]. Nevertheless the opinion seems to prevail that in typical numerical simulations a generic triangular billiard is ergodic with respect to Lebesgue measure.

A substantial amount of numerical results have been produced for right-angled triangular billiards. A careful examination of those data, (see, e.g., [18]), and recent numerical results [22] cast some doubt on the ergodicity of Lebesgue measure in these systems. In fact, rightangled billiards are closely related to symmetric billiards if one uses a Zemlyakov-Katok construction to unfold the billiard dynamics [23]. Hence we focus here on the symmetric case $\alpha=\beta$ and study the ergodic properties of 
the uniform invariant distribution by numerical means.

A necessary condition for the ergodicity of the measure $\mu$ is a vanishing Cesaro limit of the correlation function, i.e., $\sum_{n=0}^{N-1} C_{f f}(n) / N \rightarrow 0$ as $N \rightarrow \infty$. Thus, in order to measure ergodicity of the uniform distribution we introduce the order parameter

$$
\Phi_{N}=\frac{1}{N} \sum_{n=0}^{N-1}\left\langle f \cdot f \circ T^{n}\right\rangle_{\text {Leb. }},
$$

which is well known from solid state physics, measuring spontaneous symmetry breaking in phase transitions. If Lebesgue measure is ergodic then $\lim _{N \rightarrow \infty} \Phi_{N}=\langle f\rangle_{\text {Leb. }}^{2}$. Restricting to observables with vanishing Lebesgue average, $\langle f\rangle_{\text {Leb. }}=0$, one can disprove ergodicity of Lebesgue measure by showing that $\Phi_{N}$ does not vanish as $N \rightarrow \infty$.

For our numerical studies we use the observable $f=$ $\sin (2 \pi s / L)$ with $L$ the perimeter of the triangle. This observable encodes the position on the boundary and has vanishing Lebesgue average. We consider a symmetric triangle with $\alpha=\beta$ and compare findings to cases with slightly distorted symmetry, $\beta=\alpha-\pi \varepsilon$ with $\varepsilon>0$. Our findings do not substantially depend on the particular value of $\alpha$. For numerical evaluation of the correlation in Eq.(2) we use the FFT-based method described above, now with a uniform random ensemble of initial conditions.

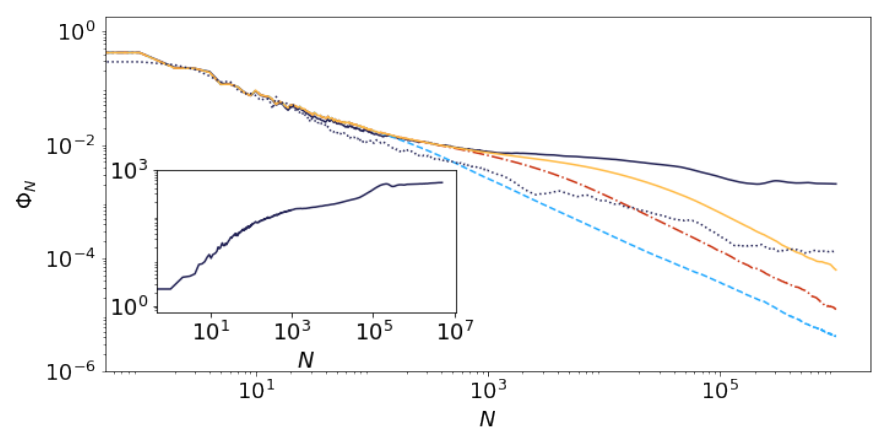

FIG. 2. Order parameter $\Phi_{N}$, see Eq.(2), on a doublelogarithmic scale for a symmetric triangle (dark blue, solid dark grey) with $\alpha=\beta=\pi(\sqrt{5}-1) / 4$ and asymmetric triangles with $\beta=\alpha-\pi \varepsilon$ with $\varepsilon=10^{-3}$ (dashed light blue), $10^{-5}$ (dash-dotted red), and $10^{-7}$ (yellow, solid light grey), along with the right-angled triangle $\alpha=\pi(\sqrt{5}-1) / 4, \beta=\pi / 2$ (dotted dark blue/grey). The correlation functions have been computed via a Fourier transform of time series of length $2^{27}$. The ensemble average has been computed using $10^{4}$ randomly generated initial conditions. The inset shows $1 / \Phi_{N}$ for the symmetric case as a function of $N$ on a double-logarithmic scale.

Fig. 2 shows the dependence of the order parameter on $N$. For slightly asymmetric triangles the order parameter tends to zero, and this tendency becomes stronger with increasing distortion. These findings support ergodicity of Lebesgue measure in asymmetric cases. In the symmetric case, results are not fully conclusive. The order parameter has no clear limit, it may either tend to a finite value or it may tend towards zero in an extremely slow fashion, see inset of Fig. 2. The data support ergodicity breaking of Lebesgue measure or, at least, point towards a very slow sub-logarithmic time scale which is not amenable to direct simulations. Above all, the findings for the symmetric case clearly differ from the asymmetric case where the order parameter algebraically converges to zero.

In order to shed more light on the ergodicity of Lebesgue measure, we evaluate the distribution of finite time ergodic averages

$$
P_{N}(z)=\left\langle\delta\left(z-\bar{p}_{N}\right)\right\rangle_{\text {Leb. }}
$$

where

$$
\bar{p}_{N}=\frac{1}{N} \sum_{n=0}^{N-1} p \circ T^{n}
$$

denotes the average of momentum. Properties of the distribution (3) may help to identify different ergodic components of the system. Numerical results in the cases of symmetric and distorted asymmetric triangles are shown in Fig. 3. The distorted asymmetric triangle shows scaling of the distribution according to large deviation theory with $P_{N}(z) \sim \exp (-N \phi(z))$ where the maximum and variance follow a law of large numbers with exponential tails. Again, the symmetric triangle is vastly different: the distribution (3) shows almost no scaling with $N$. This could point towards a flat non-equilibrium potential and many ergodic components which the uniform distribution is composed of.

In order to illustrate the strange ergodic behaviour of symmetric triangular billiards we finally evaluate the actual point-wise convergence of individual ergodic averages, see eq.(4), for a given initial value $\left(s_{0}, p_{0}\right)$. Fig. 4 shows the convergence of the ergodic average of momentum. For an asymmetric or slightly distorted billiard one finds convergence of the ergodic average to the analytic value $\langle p\rangle_{\text {Leb. }}=0$, in line with ergodicity of the uniform distribution. In stark contrast, in the symmetric case, ergodic averages may not even converge and a meandering on exponentially long time scales appears to prevail.

Conclusion We have provided compelling numerical evidence that the symmetry of triangular billiards plays a crucial role for the ergodic properties of the dynamics. While for typical irrational triangles, correlations with respect to Lebesgue measure appear to decay, the uniform distribution does not even appear to be ergodic in isosceles irrational triangles.

The role of symmetry can be convincingly demonstrated when comparing results from a symmetric triangle with a corresponding right-angled triangle. If one unfolds the dynamics of a right-angled triangle at one of the catheti one obtains the dynamics in a symmetric triangle with an almost two-to-one correspondence between 

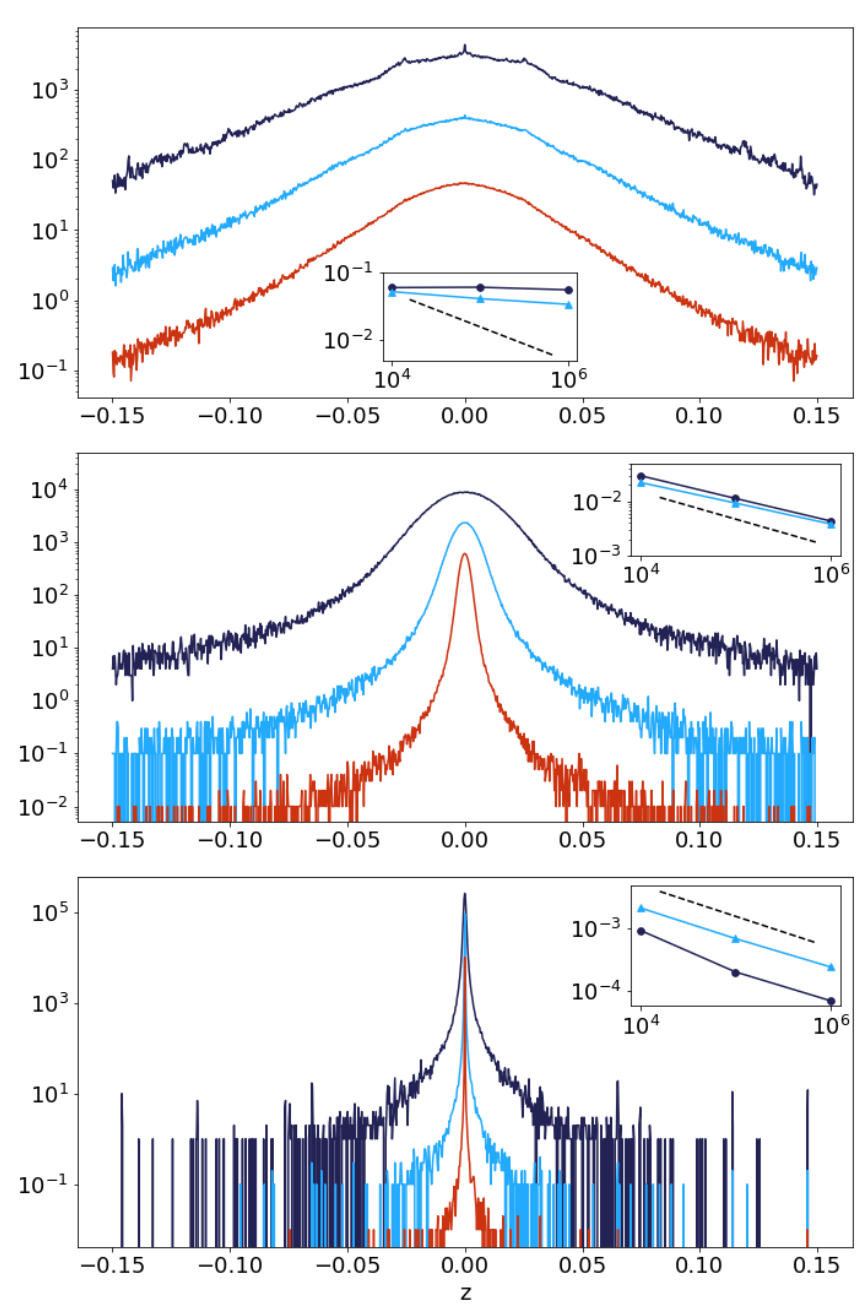

FIG. 3. Distribution of the finite time ergodic average of momentum $p$, see eq.(3), on a semi-logarithmic scale. Top: symmetric triangle with $\alpha=\beta=\pi(\sqrt{5}-1) / 4$, middle: distorted symmetric triangle with $\beta=\alpha-\pi 10^{-3}$, bottom: right-angled triangle with $\alpha=\pi(\sqrt{5}-1) / 4, \beta=\pi / 2$. Top lines (dark blue) correspond to $N=10^{4}$, middle lines (light blue) to $N=10^{5}$, and bottom lines (red) to $N=10^{6}$. Data have been computed from a uniform random ensemble of initial conditions with ensemble size $10^{6}$. The distributions have been generated as a histogram with bin size $4 \times 10^{-4}$. The insets show the halfwidth (dark blue circles) and standard deviation (light blue triangles) for the three values of $N$, along with the trivial scaling $1 / \sqrt{N}$ (dashed).

the orbits of both systems [23]. While there is no obvious relation between the ergodic properties of both systems one would expect that the dynamics in both triangles is closely related. However, if we perform the preceding simulations for a right-angled triangle then the signatures of a non ergodic Lebesgue measure seem to disappear, as already reported in [18], where evidence for weak mixing has been found. No anomalous dependence in the convergence of ergodic averages seem to be visible (see

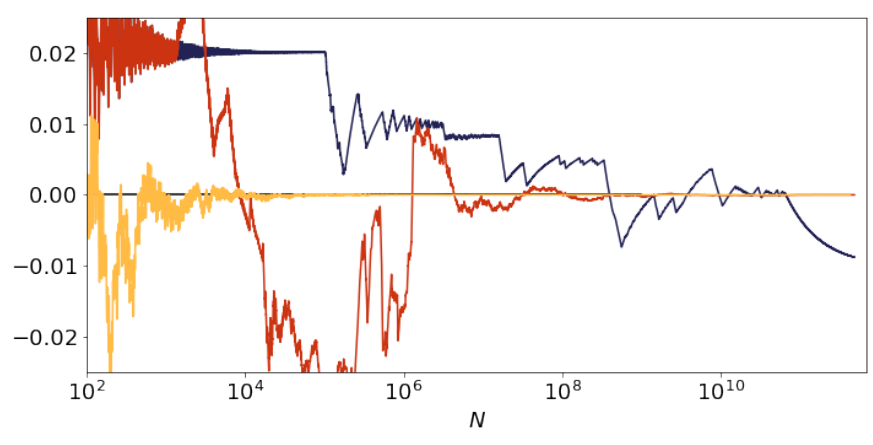

FIG. 4. Ergodic averages, see eq.(4), on a semi-logarithmic scale, for a symmetric triangle with $\alpha=\beta=\pi(\sqrt{5}-1) / 4$ (dark blue, dark grey), distorted triangle with $\beta=\alpha-\pi 10^{-7}$ (red, mid-grey), and a right-angled triangle with $\alpha=\pi(\sqrt{5}-$ $1) / 4, \beta=\pi / 2$ (yellow, light grey) for fixed initial condition $\left(s_{0}, p_{0}\right)=(0.5,0.64)$.

Fig. 4), the order parameter scales in a normal way (see Fig. 2) and the distribution of finite time averages shows a scaling which is broadly in line with the behaviour in asymmetric triangles (see Fig. 3). Hence, at least the pronounced anomalous behaviour of the symmetric case does not show up in the corresponding right angled triangle and the strange relaxation behaviour can be attributed to the symmetry of the system, since a very closely related asymmetric case does not share such a feature.

At first glance the difference between the dynamics in symmetric and right-angled triangles is rather striking. The Zemlyakov-Katok unfolding alluded to earlier maps each orbit of the symmetric triangle as well as its mirror image to an orbit of the corresponding rightangled triangle. Furthermore, time averages of symmetric observables in the symmetric triangle are also mapped onto time averages of orbits in the right-angled triangle. However, this two-to-one mapping cannot be easily inverted. While it is numerically possible to reconstruct orbits and averages of the symmetric triangle from orbits of the right-angled triangle, the corresponding procedure requires tracking the entire orbit (except, perhaps, for observables respecting the symmetry of the triangle). As the dynamics in polygonal billiards exhibits long-time correlations, this construction does not yield ordinary ergodic averages and hence the dynamics in both cases may differ for observables not respecting the symmetry of the underlying triangle, as e.g., for the data in Fig. 4. Furthermore, even the existence of a one-to-one mapping between orbits of two given dynamical systems, or more precisely a topological conjugacy, does not entail any relation between ergodic properties and time averages of the two systems. As an example, [28] shows that the tent map with constant slope, constant invariant density, and fast correlation decay is conjugate to the Farey map (see e.g., [29] for the explicit calculation) which has a marginally unstable fixed point, displays intermittency 
and ageing [30], and does not even have a well-defined invariant density. Hence, there is no a priori internal contradiction that ergodic properties in symmetric triangles may substantially differ from those in right-angled triangles.

The potential non-ergodicity of Lebesgue measure has been pointed out recently [22] without making any reference to the underlying symmetry of the system. The importance of symmetry is also mirrored by a toy map modelling billiard dynamics [20]. This model shows features similar to our findings when cases with and without symmetry are compared. Finally, symmetry turns out to be relevant when rational billiards are considered and where better analytical insight can be gained. While the uniform distribution is not ergodic in these cases, one observes very slow convergence of ergodic averages when isosceles rational triangles with large denominators are investigated. All in all, these findings support the claimed dichotomy between symmetric and asymmetric billiards.

The matter turns out to be much more complex when comparing the numerical findings with the few existing rigorous results. The author of [14] provides an explicit construction of certain irrational billiards with ergodic Lebesgue measure, and these cases may cover certain symmetric billiards as well. However, the numerical values for the angles have rather peculiar number theoretic properties and hence these values may not typically be encountered in actual numerical simulations. Therefore, these rigorous results may not be in conflict with our numerical findings. The situation is comparable to the seminal statement in [13] that typical irrational billiards have an ergodic uniform distribution. Here, typicality is understood in a topological sense, but it remains an open problem whether this means that such angles constitute a set of positive Lebesgue measure [7], let alone of full measure. Even though the question of ergodicity of the uniform distribution in isosceles triangular billiards cannot be answered currently, the stark difference of the relaxation dynamics and time scales in symmetric and asymmetric billiards is beyond any doubt a distinctive effect of the symmetry.

This still leaves us with the question of what mechanism may be at work producing the strange dynamical signatures in isosceles triangular billiards. One may take some inspiration from interval exchange transformations, a class of maps which occur in the study of rational billiards, but which also capture properties of general parabolic dynamical systems. The explicit construction given in [24] results in maps which are minimal, i.e., every orbit is dense, but which have more than one ergodic invariant density. This counterintuitive property points to a strange dynamics where dense orbits meet the different ergodic components, resulting in an exponential proliferation of the relaxation process. The exponential proliferation of quasi-stationary periods for the time aver- ages visible in Fig. 4 is also known from ageing dynamics. This phenomenon is remarkably similar to that found in stable heteroclinic networks $[25,26,31]$, where the dynamics is dominated by exponentially increasing sticking times to saddle points. In these cases symmetry plays a crucial role as well. Polygonal billiards lack an obvious hyperbolic structure which underlies the heteroclinic switching between hyperbolic saddles. However, there exists analogous phenomena in simple one-dimensional doubly intermittent maps [32]. These rigorous studies emphasise again that topological features such as dense orbits do not exclude the occurrence of strange ergodic behaviour which resembles ageing dynamics and which is visible in symmetric triangular billiards (see Fig. 4). Even though we are currently lacking a deeper analytical understanding for the phenomena occurring in symmetric billiards, let alone a rigorous account, the analogies just outlined may point towards a sophisticated heteroclinic mechanism causing the ageing dynamics in certain symmetric triangular billiards. On a related note, the role of symmetry in preventing mixing (and that of asymmetry in causing it) has been proven for typical minimal locally Hamiltonian flows, see [27] and references therein.

Without doubt, the apparent simplicity of polygonal billiards belies the fact that their dynamics is counterintuitive and their study a major challenge, with correlation decay and ergodicity wide open questions. They may serve as a testing ground for contemporary approaches in dynamical systems theory, and may well develop into a new paradigm for complex dynamical behaviour.

The authors gratefully acknowledge the support of the research through EPSRC grant EP/R012008/1. This research utilised Queen Mary's Apocrita HPC facility, supported by QMUL Research-IT. http://doi.org/10.5281/zenodo.438045

* K.Zahradova@qmul.ac.uk

[1] J. P. Eckmann and D. Ruelle, Ergodic theory of chaos and strange attractors, Rev. Mod. Phys. 57, 617 (1985).

[2] Dynamical Systems I-VIII. Encyclopedia of Mathematical Sciences, edited by D. V. Anosov, V. I. Arnold, Ya. G. Sinai, S. P. Novikov (Springer, Berlin, Heidelberg, New York, 1988-1993).

[3] Laws of Chaos: Invariant measures and Dynamical Systems in One Dimension by A. Boyarsky and P. Gora (Birkhäuser, Basel, 1997).

[4] Dynamical Zeta Functions and Dynamical Determinants for Hyperbolic Maps by V. Baladi (Springer, 2008).

[5] S. Gouëzel, Central limit theorem and stable laws for intermittent maps, Prob. Theor. Rel. Fields 128, 82 (2004).

[6] Anomalous Transport: Foundations and Applications, edited by R. Klages, G. Radons, and I. M. Sokolov (Wiley-VCH, Weinheim, 2008).

[7] E. Gutkin, Billiard dynamics: An updated survey with the emphasis on open problems, Chaos 22, 026116 (2012). 
[8] M. Keane, Interval exchange transformations, Math. Z. 141, 25 (1975).

[9] M. Boshernitzan, A condition for a minimal interval exchange transformation to be uniquely ergodic, Duke J. Math. 52, 723 (1985).

[10] Y. G. Sinai and C. Ulcigrai, Weak mixing in interval exchange transformations of periodic type, Lett. math. Phys. 74, 111 (2005).

[11] A. Katok, Interval exchange transformations and some special flows are not mixing, Isr. J. Math. 35, 301 (1980).

[12] E. Gutkin and A. Katok, in Holomorphic Dynamics, edited by X. Gomez-Mont, J. A. Seade, and A. Verjovski (Springer, Berlin, 1988), p. 163.

[13] S. Kerckhoff, H. Masur, and J. Smillie, Ergodicity of billiard flows and quadratic differentials, Ann. Math. 115, 293 (1986).

[14] Y. B. Vorobets, Ergodicity of billiards in polygons: explicit examples, Russ. Math. Surv. 51, 756 (1996).

[15] E. Gutkin, Billiards in polygons: Survey of recent results, J. Stat. Phys. 83, 7 (1996).

[16] H. Masur and S. Tabachnikov, in Handbook of Dynamical Systems, Vol 1A, edited by B. Hasselblatt and A. Katok (North Holland, Amsterdam, 2002), p. 1015.

[17] M. Viana, Ergodic theory of interval exchange maps, Rev. Mat. Comp. 19, 7 (2006).

[18] R. Artuso, G. Casati, and I. Guarneri, Numerical study on ergodic properties of triangular billiards, Phys. Rev. E 55, 6384 (1997).

[19] G. Casati and T. Prosen, Mixing property of triangular billiards, Phys. Rev. Lett. 83, 4729 (1999).

[20] G. Casati and T. Prosen, Triangle map: A model of quantum chaos, Phys. Rev. Lett. 85, 4261 (2000).
[21] M. Horvat, M. D. Esposti, S. Isola, T. Prosen, and L. Bunimovich, On ergodic and mixing properties of the triangle map, Physica D 238, 395 (2009).

[22] J. Wang, G. Casati, and T. Prosen, Nonergodicity and localization of invariant measure for two colliding masses, Phys. Rev. E 89, 042918 (2014).

[23] A. N. Zemlyakov and A. B. Katok, Topological transitivity of billiards in polygons, Math. Notes Acad. Sci. USSR 18, 760 (1975).

[24] M. Keane, Non-ergodic interval exchange transformations, Isr. J. Math. 26, 188 (1977).

[25] M. Krupa, Robust heteroclinic cycles, J. Nonl. Sci. 7, 129 (1997).

[26] P. Ashwin and P. Chossat, Attractors for robust heteroclinic cycles with a continua of connections, J. Nonl. Sci. 8103 (1998).

[27] C. Ulcigrai, Absence of mixing in area-preserving flows on surfaces, Ann. Math. 173, 1743 (2011).

[28] W. Parry, Symbolic dynamics and transformations of the unit interval, Trans. Am. Math. Soc. 122368 (1966).

[29] Y. G. Shi, A Note on the conjugacy from Farey map to the skew tent map, Bull. Malay. Math. Sci. Soc. 42649 (2019).

[30] E. Barkai, Aging in subdiffusion generated by a deterministic dynamical system, Phys. Rev. Lett. 90104101 (2003).

[31] D. Hansel, G. Mato, and C. Meunier, Clustering and slow switching in globally coupled phase oscillators Phys. Rev. E 483470 (1993).

[32] M. Blank and L. Bunimovich, Multicomponent dynamical systems: SRB measures and phase transitions Nonlin. 16 387 (2003). 